Diabetologe 2020 - 16:619-621

https://doi.org/10.1007/s11428-020-00652-5

() Springer Medizin Verlag GmbH, ein Teil von Springer Nature 2020

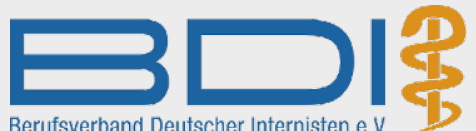

Berufsverband Deutscher Internisten e.V.

$$
\text { Gemeinsam sind wir Arzt. }
$$

Redaktion

Prof. Dr. med. Hans Martin Hoffmeister, Wiesbaden (v.i.S.d.P.)

Korrespondenzadresse

Berufsverband Deutscher Internisten e.V.

Schöne Aussicht 5

65193 Wiesbaden

Tel.: 0611 18133-0

Fax: 0611 18133-50

Folgen Sie uns auf

info@bdi.de |www.bdi.de

f www.facebook.com/lhr.BDI

twitter.com/BDI_eV

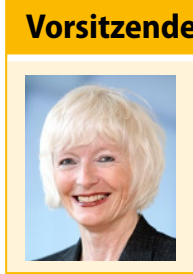

Dr. med. Cornelia Jaursch-Hancke

DKD HELIOS Klinik Wiesbaden

Aukammallee 33, 65191 Wiesbaden

\section{Partnerschaft sieht anders aus}

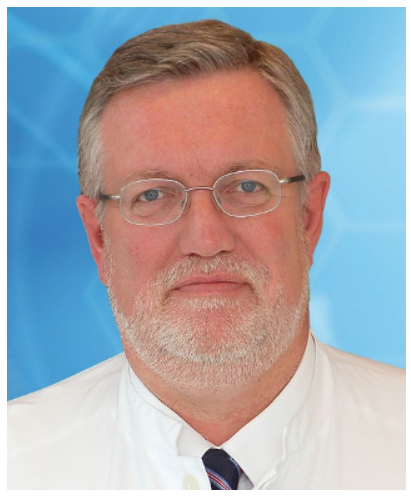

Liebe Kolleginnen und Kollegen,

gerade in Zeiten der CoronaPandemie hatte der BDI das Gespräch mit dem Verband der privaten Krankenversicherung gesucht, um über mögliche Unterstützungsmaßnahmen der Ärzteschaft im Rahmen der Corona-Krise zu sprechen. Immerhin haben wir in Deutschland ein duales Krankenversicherungssystem und die Kassenärztlichen Vereinigungen aus dem Bereich der gesetzlichen Krankenversicherung hatten doch einige Initiativen ergriffen. Hier hatte sich in der Ärzteschaft viel solidarisches Verhalten gezeigt.
Die KVen hatten sich neben anderem um Support und Schutzdies in der Anfangsphase auch etwas spärlich manchmal ausfiel. Der Verband der privaten Krankenversicherer reklamiert im Gesundheitsversicherungssystem für sich, ein systemrelevanter Player zu sein. Während der Krise nahm die PKV dankend weiter die unabhängig vom Einkommen strömenden Beiträge an. Die gleichzeitige Zurückhaltung von Patienten für Praxis- und Krankenhausbehandlung reduzierte die Ausgaben und führte zu einer deutlichen Einnahmeverbesserung.

Zwar gab es dann vereinzelte Kontakte zwischen PKV-Verband und Bundesärztekammer einschließlich finanzieller Förderung, insgesamt waren die seitens der PKV ergriffenen Maßnahmen aber sehr beschränkt und für die einzelnen „Helden der Versorgung" kein sichtbares Signal der Solidarität. Gerade aber die Ärzteschaft, die sich in den letzten Jahren bei der Diskussion um die Vereinheitlichung des Versicherungssystems in Deutschland klar zugunsten ausrüstung bemüht - wenngleich eines dualen Versicherungssystems positioniert hatte, wurde hier seitens der PKV quasi „im Regen" stehen gelassen. Die Ärzteschaft hätte sich, nicht zuletzt auch in Anbetracht ihrer Unterstützung des PKV-Systems, ein anderes Zeichen gewünscht. Partnerschaft im Gesundheitswesen sieht jedenfalls anders aus.

Prof. Dr. med. Hans Martin Hoffmeister Präsident BDI e.V.

\title{
Spahn geht Bewertungsverfahren an
}

Der Gesundheitsminister will mehr Tempo in die GBA-Verfahren bringen. Damit könnte auch die unterschiedliche Handhabung neuer Behandlungsmethoden in stationärer und ambulanter Versorgung ein Ende haben.

Neue Untersuchungs- und Behandlungsmethoden (NUB) sollen künftig schneller in die Regelversorgung kommen. Um das zu erreichen, greift Gesundheitsminister Jens Spahn (CDU) zum bewährten Mittel einer Verordnung.

Mit der „Methodenbewertungsverfahrensverordnung “ (MBVerf) soll das NUB-Bewertungsverfahren im Gemeinsamen Bundesausschuss (GBA) beschleunigt und weiterentwickelt werden. Hier gab es in der Vergangenheit aus verschiedenen Gründen berechtigte Kritik an der Arbeitsweise des GBA. Vermisst wurde u.a. die Einbindung von Berufsverbänden bei der Ersteinschätzung, eine eindeutige Definition der evidenzbasierten Medizin bei der
Beschlussfassung und in den tragenden Gründen, vor allem aber die Berücksichtigung von Versorgungsnotwendigkeiten von Verfahren mit niedriger Evidenz.

\section{》) Für Praxen oft nicht nutzbar}

Kritisiert wurde insbesondere die oft zu lange Verfahrensdauer, die dazu führen konnte, dass die Entscheidung zu einem Zeitpunkt getroffen wurde, wo die evaluierte Methode schon vom medizinischen Fortschritt überholt worden war. Zusammen mit dem unterschiedlichen Leistungsrecht, nämlich dem Erlaubnisvorbehalt im ambulanten und dem Verbotsvorbehalt im stationären Sektor, führte dies dazu, dass sinnvolle Untersuchungsund Behandlungsmethoden nur im Krankenhaus zur Verfügung standen, obwohl sie auch hätten ambulant erbracht werden können. Der Verordnungsentwurf könnte tatsächlich zu einer qualitativ verbesserten ambulanten Versorgung führen. Eine Analy- 
se des Entwurfs aus dem Hause Spahn:

$\$ 2$ Antrag: Die Antragstellung wurde nicht verändert. Sie bleibt den unparteiischen Mitgliedern des GBA, der Kassenärztlichen Bundesvereinigung (KBV), dem Spitzenverband der Krankenkassen und der Deutschen Krankenhausgesellschaft (DKG) oder den Vertretern der Krankenhäuser vorbehalten. Fachgesellschaften und Verbände können somit weiter nur über diese Personen und Körperschaften und über die Vertreter der Krankenhäuser Vorschläge einbringen. Deren Entscheidung, ob sie den Vorschlag zur Beratung übernehmen, ist dabei meist intransparent. Es wäre wünschenswert, wenn die Träger des GBA ihre Entscheidung und deren Gründe auch im Vorfeld transparent machen müssten.

Ob der Antrag beraten wird, soll der GBA laut Verordnungsentwurf dann binnen drei Monaten entscheiden.

$\$ 3$.Ankündigung der Bewertung und Einholung einer Ersteinschätzung: Die Annahme des Antrags und des Beratungsthemas muss umgehend veröffentlicht werden. Dabei dürfen auch weitere Sachverständige einschließlich der Verbände von Leistungserbringern Stellungnahmen abgeben. Sie haben dafür mindestens einen Monat Zeit.

$\$ 4$ Ermittlung und Auswertung der vorliegenden Erkenntnisse: Sowohl für den Ausschuss selbst als auch für die eventuell beauftragten wissenschaftlichen Institutionen wie z. B. das Institut für Qualität und Wirtschaftlichkeit im Gesundheitswesen (IQWiG) muss spätestens nach einem Jahr der Bericht vorliegen. Dies gilt auch für die Präzisierung der Entscheidung nach den in der Verordnung beschriebenen Kriterien der evidenzbasierten Medizin.

$\$ \$ 5,6,7$ Bewertung und Beschlussfassung: Der GBA erhält für seine Stellungnahme ebenfalls drei Monate Zeit. Die Frist läuft, nachdem der Bericht (sie- he Paragraf 4) vorliegt. Bis $\mathrm{zu}$ diesem Punkt der Entscheidung sind somit maximal 18 Monate seit dem veröffentlichten Antrag vergangen, im Vergleich zu den bisherigen Regelungen, die einen Zeitrahmen von zwei Jahren vorsahen, insgesamt eine deutliche Verkürzung.

Der GBA hat vier Entscheidungsmöglichkeiten:

- Anerkennung der Methode.

- Hinweise auf das Potenzial als Behandlungsalternative. Sollte jedoch die hinreichende Evidenz dazu fehlen, kann eine Erprobungsrichtlinie nach Paragraf 137 e Abs. 1 und 2 SGB V veranlasst werden.

- Die Methode ist schädlich und/oder unwirksam.

- Aussetzen der Entscheidung für einen befristeten Zeitraum, da in naher Zukunft wissenschaftliche Ergebnisse zu erwarten sind, die eine Entscheidung ermöglichen.

Droht eine unzumutbare Verlängerung des Verfahrens durch die Entscheidungsmöglichkeiten, haben die unparteiischen Mitglieder nach weiteren sechs Monaten einen eigenen Entscheidungsvorschlag vorzulegen. Damit werden 36 Monate in der Regel in der Entscheidungspraxis nicht überschritten.

Bei der dann folgenden Beschlussfassung wird dem unterschiedlichen Leistungsrecht ambulant und stationär zusätzlich Rechnung getragen, bei der die stationäre Leistung solange erbringbar ist, wie eine negative Entscheidung im GBA nicht getroffen wurde. Nach Paragraf 7 Abs. 3 muss nach dem Entwurf nämlich entschieden werden, ob diese Praxis weiter erlaubt ist oder ob das Verfahren auch im Krankenhaus in Zukunft nicht mehr zur Verfügung stehen wird. Damit würden die Folgen des unterschiedlichen Leistungsrechtes reduziert, in dem auch unwirksame oder schädliche Verfahren in der Krankenhausversorgung nicht mehr zugelassen sind.

S8Tragende Gründe: Bei den tragenden Gründen des anschließenden GBA-Beschlusses muss die Versorgungsrealität genauso berücksichtigt werden, wie die Tatsache, dass alternative Behandlungsverfahren nicht zur Verfügung stehen, damit die ambulante Versorgung nicht qualitativ gefährdet wird. Damit können z. B. diagnostische Verfahren, die für bestimmte sinnvolle Behand-

lungen erforderlich sind, trotz fehlender Evidenz vom Gemeinsamen Bundesausschuss positiv entschieden werden.

\section{Dr. med. Hans-Friedrich Spies}

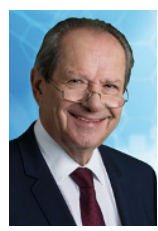
Vorstandsmitglied des BDI

\section{Chance statt Risiko: Mit Daten Leben retten}

Digitalisierungsexperten der Union wollen den Nutzen der E-Patientenakte erhöhen. Die Bürger sollen für eine bessere Medizin nicht nur ein Recht auf eine Datenspende erhalten. Die Akte soll ihnen auch direkt Infos zu neuen Behandlungsmethoden liefern.

Pünktlich zur Debatte ums Patientendatenschutz-Gesetz (PDSG) und den damit einhergehenden Regelungen zur elektronischen Patientenakte (ePA) haben sich nun Gesundheitsund Digitalisierungsexperten der CDU/CSU-Fraktion im Bundestag zu Wort gemeldet. In einem gemeinsamen Positionspapier stellen sie fest, dass die Digitalisierung im Gesundheitswesen aufgrund regional uneinheitlicher Rechtsvorgaben, aber auch wegen einer „risikobeschwerten“ Debattenkultur in Deutschland regelmäßig ins Stocken gerät.

》) Im Einklang mit Gesundheitsweisen

In ein ähnliches Horn stieß vor wenigen Monaten erst der Sachverständigenrat zur Begutachtung und Entwicklung im Gesundheitswesen als er mahnte, dass die Chancen und Risiken der Digitalisierung immer sorgsam gegeneinander abgewogen werden müssten und Daten- schutz kein Selbstzweck sei (wir berichteten).

Die Autoren des Papiers mit dem Titel „Mit Daten Leben retten: Für eine bessere Patientenversorgung durch Digitalisierung und Gesundheitsforschung " lassen keinen Zweifel daran, dass die Bürger ein uneingeschränktes Recht auf datenschutzrechtliche Selbstbestimmung haben. Das beinhalte sowohl die Entscheidung, Daten der Wissenschaft vorzuenthalten als auch das Recht, Daten der Forschung zu spenden. Dieses Recht dürfe nicht beschnitten werden. Denn Datenspenden böten eine „Chance, Krankheiten durch eine enge Vernetzung von Arzt, Patient und Forschung effektiver zu bekämpfen“. Die Politik müsse auch diesem Wunsch der Bevölkerung gerecht werden. Insbesondere da die Mehrheit der Deutschen in Umfragen angibt, ihre Daten der Wissenschaft zur Verfügung stellen zu wollen.

Deswegen fordern sie, die Möglichkeit einer Datenspende für Forschungszwecke in der E-Patientenakte schneller als geplant zu verankern. Bislang sollte diese Funktion erst ab 2023 möglich sein.

Weiterhin machen sie sich für eine Harmonisierung des Datenschutzes auf Bundesebene stark, damit Forschungsvorhaben auch länderübergreifend 
möglich werden und nicht mehr an restriktiven datenschutzrechtlichen Regelungen einzelner Bundesländer scheiterten. Im Rahmen dessen sei auch die Einrichtung einer nationalen Koordinierungsinstanz zu prüfen, damit eine verlässliche und deutschlandweit funktionierende Dateninfrastruktur geschaffen werden könne.

Die Nutzung der Daten sollte auch nicht, wie bislang im PDSG vorgesehen, auf die Universitäten beschränkt bleiben, sondern auch der in Deutschland ansässigen Gesundheitswirtschaft möglich sein. Dies wäre sowohl im Interesse der Patienten als auch im Sinne des Wirtschaftsstandortes Deutschland. Damit mit den Daten auch innovative und sinnvolle Forschung möglich ist, müsse zudem sichergestellt werden, dass der Forschungsdatensatz qualitativ hochwertig und unter ethisch und rechtlich einwandfreien Bedingungen entstanden sei. Hierzu müssten die Akteure im Gesundheitswesen positiv incentiviert werden - finanziell wie mit Know-how. Der Weg, die Digitalisierung mit Sanktionen voranzutreiben, wie bei den TI-Konnektoren, hätte sich nämlich als unwirksam erwiesen.

Besonders interessant ist, dass die Autoren davon ausgehen, dass die Nutzung der pseudonymisierten Versorgungsdaten der Schlüssel zu einer individualisierten und auf den neuesten Erkenntnissen beruhenden $\mathrm{Pa}$ tientenversorgung ist. Wer bis jetzt dachte, der Datenfluss solle einer Einbahnstraße gleichen - vom Patienten zum Forscher hat sich getäuscht. Nein, in den Augen der Politiker muss die
ePA „zugleich ermöglichen, dass neue Erkenntnisse aus der Forschung direkt den Patienten erreichen können." Nur so könne personalisierte Medizin Realität werden.

Konkret könnte das also heißen, dass ein Patient aufgrund der in seiner ePA abgespeicherten Daten Hinweise zu neuen oder ggf. noch nicht ausgeschöpften Behandlungsoptionen erhält. Technisch ist so etwas theoretisch möglich, ohne dass patientenspezifische Infos zentral verarbeitet werden müssen. Auch wenn eine solche Funktion viele Chancen und Vorteile bringen könnte, hätte sie einen erheblichen Einfluss auf das Arzt-Patienten-Verhältnis und würde zumindest in dieser Form die Übermittlung von medizinischen Informationen entmenschlichen.

\) Auswirkung aufs Arztverhältnis

Neu ist auch die Idee, die ePA dahingehend weiterzuentwickeln, dass ein Austausch von Patienten untereinander möglich wird.

Das Positionspapier hat ganz offensichtlich das Ziel, dem Entwurf des PDSG einen noch etwas progressiveren Anstrich zu verpassen. Daher ist es wichtig, dass der BDI hier in einen konstruktiven Dialog geht und seine Expertise einfließen lässt.

\section{Dr. med. Kevin Schulte}

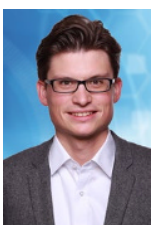
Vorstandsmitglied der außerordentl. Mitglieder des BDI Sprecher Junges Forum im BDI

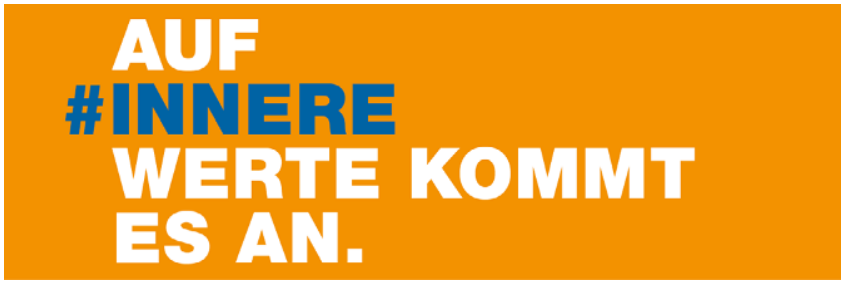

Der BDI bietet Ihren Patienten seriöse Informationen über das Virus. Millionen Patienten nutzen die Plattform bereits.

Das Coronavirus SARS-CoV-2 ist noch weitgehend unbekannt, umso größer ist der Informationsbedarf an Meldungen aus seriösen Quellen. Der BDI veröffentlicht auf seiner Patientenplattform www.internistenimnetz.de aktuelle Meldungen aus Forschung und Praxis über das Virus, einen Übersichtsartikel zum Krankheitsbild Covid-19 sowie einen CoronaTicker mit täglich neuen Meldungen der Deutschen Presse Agentur (dpa) über die Auswirkungen der Pandemie auf Alltag und Gesellschaft. Außerdem wurden wichtige Hotlines und Adressen mit Patienteninformationen und die hauptsächlichen Symptome von Covid-19 im Vergleich zu Erkältung und Grippe auf der Startseite zusammengestellt.

All diese Informationen $\mathrm{zu}$ Corona werden auch direkt in die PraxisApp „Mein Internist“ eingespeist, die der BDI für die Kommunikation zwischen $\mathrm{Pa}$ -
tientInnen und InternistInnen falls erwünscht auch mit Chat und Videofunktion (inkl. Zertifizierung, die vor dem Abschluss steht) - realisiert hat, ohne dass die PatientInnen dazu in die Praxis kommen müssen. So können unnötige Termine in der Praxis gerade in Zeiten einer sich ausbreitenden Pandemie vermieden werden.

Die Freischaltung der PraxisApp „Mein Internist“ ist ganz einfach und kann online unter www.monksaerzteimnetz.de/ praxisapp/anmeldung/ beantragt werden. Sie sollten dann auch Ihre eigenen PatientInnen über das neue Angebot informieren, die sich bei Interesse die PraxisApp „Mein Internist“ kostenlos im App Store (iPhone) oder bei Google Play (Android Smartphone) herunterladen können, den eigenen Arzt aus der Arztliste auswählen und sich registrieren.

Für weitere Informationen wenden Sie sich gerne direkt an: info@monks-aerzte-im-netz.de Tel: 089 642482-12

(eb) 